\title{
Comparing Output from Two Methods of Participatory Design for Developing Implementation Strategies: Traditional Contextual Inquiry vs. Rapid Crowd Sourcing
}

\section{Emily Becker-Haimes ( $\nabla$ embecker@upenn.edu )}

University of Pennsylvania https://orcid.org/0000-0002-9922-8667

\section{Brinda Ramesh}

University of Pennsylvania

Jacqueline Buck

University of Pennsylvania

Heather J. Nuske

University of Pennsylvania

Kelly A. Zentgraf

University of Pennsylvania

Rebecca E. Stewart

University of Pennsylvania

David S. Mandell

University of Pennsylvania

\section{Research}

Keywords: participatory design, evidence-based practice, implementation science, implementation strategy design

Posted Date: November 22nd, 2021

DOI: https://doi.org/10.21203/rs.3.rs-1075424/v1

License: (c) (i) This work is licensed under a Creative Commons Attribution 4.0 International License. Read Full License 


\section{Abstract}

\section{Background}

Participatory design methods are a key component of designing tailored implementation strategies. These methods vary in the resources required to execute and analyze their outputs. No work to date has examined the extent to which the output obtained from different approaches to participatory design varies.

\section{Methods}

We concurrently used two separate participatory design methods: 1) field observations and qualitative interviews and 2) rapid crowd sourcing (an innovation tournament). Our goal was to generate information to tailor implementation strategies to increase the use of evidence-based data collection practices among one-to-one aides working with children with autism. Each method was executed and analyzed by study team members blinded to the output of the other method. We estimated the personnel time and monetary costs associated with each method to further facilitate comparison.

Results

Observations and interviews generated nearly double the number of implementation strategies $(n=26)$ than did the innovation tournament $(n=14)$. When strategies were classified into clusters from the Expert Recommendations for Implementing Change (ERIC) taxonomy, there was considerable overlap in the content of identified strategies. Strategies derived from observations and interviews were more specific than those from the innovation tournament. Nine strategies $(23 \%)$ reflected content unique to observations and interviews and $4(10 \%)$ strategies were unique to the innovation tournament. Only observations and interviews identified implementation strategies related to adapting and tailoring to context; only the innovation tournament identified implementation strategies that used incentives. Observations and interviews required more than three times the personnel hours than the innovation tournament, but the innovation tournament was more costly overall due to the technological platform used.

\section{Conclusions}

There was substantial overlap in content derived from observations and interviews and the innovation tournament. However, each yielded unique information. To select the best participatory design approach to inform implementation strategy design for a particular context, researchers should carefully consider what each method may elicit and weigh the resources available to invest in the process.

\section{Trial Registration}

N/A 


\section{Contributions To The Literature}

- This study advances our understanding of the strengths and limitations of different approaches to participatory design to inform implementation strategy generation.

- The two methods of participatory design - observations/interviews and an innovation tournament yielded substantial overlap in the information obtained, but there was unique output generated by each method.

- The innovation tournament required less time investment to complete; however, its electronic platform was expensive, making its overall cost higher than that of observations and interviews

- Results can guide researchers to carefully consider what each method offers and weigh the resources available (e.g., personnel time vs. money) to invest in the process.

\section{Introduction}

Implementation strategies, the methods used to improve the use and sustainment of evidence-based practices (EBPs), are the backbone of implementation science (1). Carefully selecting implementation strategies that target theorized implementation mechanisms (2), guided by contextual inquiry to identify implementation determinants (3), is de rigueur for implementation efforts. There are several published methods for selecting and tailoring implementation strategies (2), all of which emphasize the importance both of collaborating with stakeholders, or "end-users," of the intervention being implemented, and of drawing on methods from participatory design to do so. Because implementation is context dependent, implementation strategies will be more successful if those leading implementation efforts learn from those on the front line who will use the intervention of interest. Outputs from contextual inquiry ideally identify implementation determinants, which then drive which implementation strategies strategy selection $(4,5)$.

Participatory design approaches have a long history in many fields but their adoption as a method for selecting and tailoring implementation strategies is more recent. Participatory design originated as a collaborative method of product design that engages end users in designing new products (6). Participatory design methods can include observation of product use in the field, story boarding, interviews, and focus groups (7). Implementation science has borrowed from other disciplines to add concept mapping and intervention mapping as important tools; both rely heavily on inputs obtained through rigorous contextual inquiry, using methods such as field observations and qualitative interviews $(8,9)$.

Many approaches to participatory design are time intensive and costly. It is not clear which participatory design approach an implementation scientist should pick when engaging end users in implementation strategy development for a target EBP, nor is it known whether different approaches will lead to varying results. To our knowledge, no one has compared the results of using different approaches of participatory design on the resultant implementation strategy output (10) 
This study compared output from two participatory design approaches: (1) a traditional contextual inquiry approach (i.e., field observations and qualitative interviews with stakeholders) versus (2) a more recent addition to the panoply of participatory design tools, an innovation tournament. Innovation tournaments are a more rapid approach to participatory design that involve a kind of crowd sourcing - a competition in which members of a group can submit ideas about how to address a challenge. These ideas compete with one another through several rounds of vetting. Other members of the group can vote for and comment on participants' different ideas. In the last round, experts work with the originators of the most popular and promising ideas to refine them for a last round of voting. Innovation tournaments have become particularly popular in health care and other industries (11-13). Stewart and colleagues recently used one to identify potential implementation strategies to increase the use of evidence-based practices in outpatient mental healthcare, demonstrating their feasibility and potential utility in generating tailored implementation strategies in behavioral health (14). As innovation tournaments are designed to be conducted much more rapidly and inexpensively than traditional contextual inquiry approaches, with less burden on stakeholder partners, identifying whether their output produces comparable results to those of more traditional approaches has important implications for how we use participatory design in implementation science.

Our secondary aim was to categorize the resources involved with executing and analyzing the data obtained from each participatory design approach. Given the breadth of potential implementation strategies that emerged from Stewart and colleagues' innovation tournament, we hypothesized the innovation tournament would produce output comparable to that obtained from observations and qualitative interviews, with less burden on stakeholders and research staff.

\section{Methods}

This work was conducted as part of a larger study that integrated behavioral economics and implementation science principles to increase data collection among behavioral health technicians (BHTs) working one-on-one with children with an autism spectrum disorder in schools (15). Quantitative data collection on child response to intervention and progress monitoring is a core EBP component of applied behavioral analysis (ABA) for children with autism yet is poorly implemented in practice (16). To inform implementation strategy design, our team concurrently: (1) engaged in observations of and interviews with BHTs and interviews with their supervisors and (2) conducted an innovation tournament inviting behavioral health paraprofessionals, including BHTs and their supervisors, to participate. We synthesized data obtained from each method using a systematic, iterative processes to identify implementation strategies.

To avoid cross-contamination of output from each method, the research team divided into two separate groups for the duration of data collection and synthesis so that each team was unaware of outputs or information obtained from the other method of participatory design. We briefly describe the participatory design strategy and data analysis below. The City of Philadelphia and University Pennsylvania Institutional Review Boards approved the study. 


\section{Observations and Interviews}

We recruited 9 BHTs and 7 BHT supervisors from four different agencies in Philadelphia (63\% female; $31 \%$ White; $44 \%$ Black; $6 \%$ Asian; $19 \%$ Hispanic/Latinx). Recruited BHTs had to be working with a schoolaged child with autism. BHTs allowed us to observe their data collection practices in the field and participated in a subsequent qualitative interview over the phone. Two BHTs completed a qualitative interview only due to COVID-19 social distancing restrictions put into place midway through data collection. Recruited BHT supervisors participated in phone interviews only. We obtained informed consent from each participant prior to any research activities.

Prior to observations, author HJN trained research team members in a structured observation coding system adapted to document barriers and facilitators to BHT data collection $(17,18)$. After obtaining BHT consent and formal approval from the school in which the BHT worked, research staff traveled to the BHT's work site for a one-hour field observation. Two research team members (EBH and BR) attended the first two observations to ensure reliable use of the structured field observation form; one team member completed the remaining observations and debriefed with the second team member immediately after. During each observation, research staff observed BHT data collection practices from the back of the classroom using the structured form. Research staff noted, in real time, perceived barriers to data collection they observed. At the conclusion of the one-hour observation, staff spent approximately 5 minutes asking the BHT to expand on observed barriers (e.g., "I noticed you didn't take data in X interval. What, if anything, got in the way of you doing so?"). After their observation, BHTs scheduled their qualitative interview.

Qualitative interview guides for BHTs and BHT supervisors were designed to assess data practices BHTs used, assess barriers to engaging in quantitative data collection, and elicit recommendations on how quantitative data collection could be made easier for BHTs. BHTs additionally answered standard belief elicitation questions and questions adapted from a theory-guided interview created by Potthoff and colleagues $(19,20)$. Copies of the interview guides are available upon request. Thematic saturation was reached after the 9th BHT interview and the 7th BHT supervisor interview.

The interviewer audio-recorded the interview and a member of the research team transcribed the recording. The researchers who conducted the field observation (EBH or BR) analyzed the interviews, so that information learned via observations could guide interpretation of qualitative responses. The research team developed a structured codebook via iterative review of three transcripts and applied it to the remaining transcripts. The a priori code of "barriers and facilitators to quantitative data collection" was the primary code of interest for this study. Coders met regularly to review codes and resolve discrepancies.

From the coded transcripts, we amassed a list of identified barriers across BHTs and BHT supervisors. The same two staff members systematically reviewed each identified barrier and generated one or more discrete implementation strategies that would address each barrier, drawing on their review of all 
qualitative data and field observation notes collaboratively and systematically, guided by the Expert Recommendations for Implementing Change (ERIC) taxonomy and the Behavior Change Wheel $(21,22)$. To ensure comprehensiveness, a third team member who had administered qualitative interviews but was not involved in the coding process (KAZ) then reviewed the list of barriers and independently generated corresponding implementation strategies. This third team member did not identify any additional implementation strategies. This process resulted in a final set of 26 discrete implementation strategies.

\section{Innovation Tournament}

Our innovation tournament procedure was guided by the one Stewart and colleagues used. It was developed through the Penn Medicine Center for Health Care Innovation $(12,14)$. We invited behavioral health professionals working with children with autism in Philadelphia schools $(n=21)$, including BHTs and their supervisors, who had provided us their email addresses at previous in-person site visits. We sent them email invitations to participate in an innovation tournament titled "Your Idea Matters! The Data Collection Improvement Challenge." We also asked behavioral health agency leaders $(n=27)$ and team meeting leaders at in-person site visits $(n=5)$ to forward recruitment invitations to their staff. Some paraprofessionals may have received the invitation more than once (i.e., from a direct email from our team, from their agency leader, and from their team meeting leader). We sent agency leaders a priming email approximately 2 weeks prior to the start of the tournament, as advance contact aligns with recommendations in survey design (23). We then sent the first invitation email to agency leaders, team meeting leaders, and paraprofessionals, which included the link to participate, and 5 reminder emails over the course of 5 weeks. During the second week of reminders, we contacted agency leaders to participate and invited their staff to participate by phone.

We made the web link to the innovation tournament available for 5 weeks in October 2019. We hosted the online tournament on the "Your Big Idea Platform," that facilitates crowdsourcing for ideas and solutions. Innovation challenges are posted to the platform, and participants can submit an idea and comment and rate ideas submitted by other participants. Participants opened the link and provided consent before participating.

Developing the right tournament prompt (i.e., the question for participants) is critical to the success of an innovation tournament (14). We iteratively designed the prompt for this study. The research team generated the question language via email and in weekly meetings, and then sought feedback from 2 BHTs on the wording and design of the prompt. The final prompt asked, "What would make data collection about children with autism easier, more useful, and more motivating for you?" Participants could submit and rate as many ideas as they liked.

Eleven individuals submitted 14 ideas. Procedures for reviewing, rating, identifying "winning ideas," and categorizing output followed established procedures (14). Briefly, the research team formed a Challenge Committee consisting of an expert panel of stakeholders to vote on the submissions. The committee included two city administrators, two agency stakeholders, one autism content expert, and one behavioral science expert. The committee rated the 14 ideas on their potential impact as an implementation strategy. 
Participants with the top three ideas were considered "winning participants;" these participants were later celebrated at a community event.

To categorize information shared through the innovation tournament platform into clear implementation strategies, three members of the research team (JEB, RES, DSM) met and applied aspects of the NUDGE (Narrow, Understand, Discover, Generate, Evaluate) framework to extrapolate the discrete implementation strategies from the information submitted by innovation tournament participants. See (24) for a detailed description of how the NUDGE framework is applied to analysis of innovation tournament data. Briefly, this is an iterative process of: (1) generating hypotheses about behavioral barriers that interfere with EBP delivery through structured brainstorming that links the reported barriers to core principles from behavioral science (e.g., cognitive biases and heuristic thinking), (2) de-duplicating hypotheses, and (3) rapidly validating hypothesized barriers through expert consultation, review of the literature, and confirmation by core stakeholders. This output then informs generation of tailored implementation strategies to directly address the behavioral barriers identified.

\section{Resources \& Cost of Each Method}

To capture resources involved with each method, we captured the time and costs associated with each method, guided by principles of time-driven activity-based costing, a method increasingly employed in implementation science (25). Researchers who executed each participatory design method estimated the time and money spent throughout the process of data collection and synthesis.

\section{Results}

Table 1 shows the implementation strategies that each participatory design approach generated. To facilitate comparison, the first author $(\mathrm{EBH})$ classified all implementation strategies across the two approaches using the ERIC taxonomy, using the clusters identified by Waltz and colleagues $(22,26)$. An independent reviewer (HJN) double coded $50 \%$ of all strategies, with $100 \%$ concordance. 
Table 1

Output from Innovation Tournament Compared with Observations/Qualitative Interviews

\section{Identified Implementation Strategies}

Implementation
Strategy
Domain ${ }^{1}$

Adapt and

Tailor to

Context
Innovation

Tournament

$(n=14$ strategies $)$

None

Change

Infrastructure

Engage

None

1. Change the way

$\mathrm{BHT}$ feedback is integrated into treatment planning

2. Changes the forms or templates used to collect data

3. Digitize data collection to reduce effort

4. Integrate data collection into course of day

5. Make forms simple to use

6 . Rely on drop down menus and tallies* to do within the chaotic school environment*

2. Eliminate Wi-Fi dependency of electronic data record*

3. Ensure that data required for tracking is of behaviors that can reasonably be tracked from a short distance*

\section{Observations/Qualitative Interviews \\ ( $n=26$ strategies)}

1. Advocate for adapting the timing of treatment plan regulations to link to changes in behavior rather than calendar months

2. Consult with software developers to address limitations of current data collection capture systems in the electronic health record

3. Eliminate redundancies in data collection processes by eliminating paper forms and conducting direct data entry into the required electronic health record

4. Redesign data capture software to allow for the flexibility required to capture the range of data for specific client

5. Utilize mobile electronic data tracking platform that builds in time clocks to facilitate monitoring across environments or supply all BHTs with watches

6. Ensure that mobile electronic data tracking platforms are light and easy to transport

7. Provide supplies to ameliorate the strain caused by heavy laptops and notebooks (e.g., a small standing desk)*

8. Reposition students, teachers, and BHTs in classroom to better facilitate intervention and data collection*

9. Assess the strengths and limitations of current data collection system*
None

Consumers

Evaluative \&

Iterative

Strategies
1. Give feedback to

BHTs on treatment plans*

None 


\section{Identified Implementation Strategies}

\section{Provide Interactive Assistance \\ 1. Offer in vivo coaching}

Support Clinicians

Training \&

Educate Stakeholders visuals
2. Provide immediate feedback on data collection
1. Provide on-site coaching to BHTs on how to balance data with intervention in response to challenging behaviors

2. Enhance channels of communication between BHTs and supervisors

3. Increase the amount of supervisory support available to BHTs, particularly as it relates to direct observation in the field

4. Hold regular supervision meetings in which the BHT and supervisor troubleshoot around limitations of current data collection system*

5. Hold regular supervision meetings in which the BHT discusses examples of competing demands and/or role-plays data collection during problem behaviors*

1. Enhance perceived utility of data collection (e.g., through feedback of client progress) their data is used with

2. Provide regular reminder mechanism for clinician, e.g., hourly text message*

1. Improve training

2. Provide skills training in functional analysis
1. Conduct best data collection practices training for active and outdoor times

2. Conduct training on best practices for data collection while clients display problem behaviors

3. Include definitions of target behaviors for data collection on data record sheet

4. Provide portable educational materials to remind BHTs on best practices for collecting data on client problem behaviors

5. Provide targeted skills training to BHTs on how to balance data collection with intervention in response to challenging behaviors

6. Provide targeted training for BHTs in defining behaviors for data collection

7. Train BHTs in data collection interface, including each feature's function and use
Utilize Incentive Strategies
1. Acknowledge BHT

for collecting data*

2. Increase rewards for data collection*

None 


\section{Identified Implementation Strategies}

${ }^{1}$ Clusters from Waltz and colleagues 2015.

* Implementation strategy content not reflected in output from the opposing method.

As seen in Table 1, observations and interviews resulted in nearly double the number of implementation strategies $(n=26)$ than did the innovation tournament $(n=14)$. Both methods combined identified implementation strategies spanned eight of the nine clusters of implementation strategies identified by Waltz and colleagues (26). No implementation strategies from either method were generated in the cluster of engaging consumers (e.g., involving children and their families). Although the observations and interviews generated more strategies than the innovation tournament did, much of the additional content from observation and interviews overlapped with innovation tournament data. Specifically, when examined by implementation strategy cluster, 27 of the 40 total identified implementation strategies $(68 \%)$ reflected comparable content obtained from each method. Areas of overlap were observed in the following implementation strategy clusters: changing the infrastructure (e.g., adjusting physical structures and equipment, changing record systems), providing interactive assistance (e.g., enhancing clinical supervision), supporting clinicians (e.g., reminding clinicians), and training and educating stakeholders (e.g., enhancing training and consultation practices).

Within each of these areas, however, findings from the observations and interviews tended to yield more specific implementation recommendations than the innovation tournament did. For example, the innovation tournament identified the importance of "improving training" and "providing skills training in functional analysis." In contrast, observation and interview data yielded 7 specific recommendations to improve training in response to specific implementation barriers to data collection that BHTs reported, for example, training on best data collection practices, training for active and outdoor times, and training on best practices for data collection when clients display problem behaviors.

More than a third of identified implementation strategies were unique to one approach. In three of the nine implementation strategy clusters, only one participatory design approach generated a potential implementation strategy. Specifically, only data from the observations and interviews identified implementation strategies related to adapting and tailoring to context (e.g., promoting adaptability). Only data from the innovation tournament identified implementation strategies that used incentives or evaluative and iterative strategies.

Table 2 shows the estimated costs of each participatory design method, separated by preparation costs, data collection costs, and data synthesis costs. Overall, personnel time was substantially higher for the observations and interviews ( 263 hours) than for the innovation tournament ( 68 hours). However, the overall cost of the innovation tournament, which relied on an electronic platform to power idea submission and allow participants to comment on and rate ideas submitted by other participants, was much higher than that for observations and interviews. 
Page 11/18 
Table 2

Estimated Costs Associated with Each Participatory Design Approach Innovation Tournament Observations/Qualitative Interviews

Activity

Time

(hrs)
Personnel

Cost Activity

$(\$)$

10

Generate

innovation

Tournament

prompt

Set up "Your Big Idea" platform

10

200

200 outreach (e.g.,

agency visits,

travel costs,

communication

with potential

participants)

Recruitment

outreach (e.g.,

phone calls,

emails)
10

200

Obtain letters of

support (includes

travel to sites and

car rental fee)

Training in observation and

interview

procedures
Personnel

Time

$(\mathrm{hrs})^{\mathrm{a}}$
Cost

(\$)

Total Time \& Cost for

30

600

84

20

Preparation

Data

Collection

Operating

innovation

tournament on

the "Your Big Idea

Platform"

Innovation

tournament activities (notify

winners, set up

meetings, discuss

ideas)
12

10

200

Conduct interviews

$(n=17)$
Conduct

observations ( $n=$

$\begin{aligned} 14,594 & \text { Co } \\ & \text { ob } \\ & 7)\end{aligned}$

42

840

Interview

transcription 
Data

Synthesis

\begin{tabular}{|c|c|c|c|c|c|}
\hline $\begin{array}{l}\text { Behavioral } \\
\text { diagnostics data } \\
\text { analysis }\end{array}$ & 16 & 320 & $\begin{array}{l}\text { Develop and train } \\
\text { on qualitative } \\
\text { coding guide }\end{array}$ & 21 & 420 \\
\hline & & & Code interviews & 20 & 400 \\
\hline & & & $\begin{array}{l}\text { Analyze barriers } \\
\text { and generate } \\
\text { implementation } \\
\text { strategies }\end{array}$ & 18 & 360 \\
\hline $\begin{array}{l}\text { Total Time \& Cost for Data } \\
\text { Synthesis }\end{array}$ & 16 & 320 & & 59 & 1,180 \\
\hline Total Overall Cost & 68 & 15,714 & & 263 & 5,166 \\
\hline
\end{tabular}

\section{Discussion}

To our knowledge, this is the first effort to directly compare the output of two distinct methods of participatory design and their resultant implications for implementation strategy design. Data suggested a high degree of convergence in output across the two strategies. This suggests that use of an innovation tournament can yield information comparable to that obtained from the more traditional observations and qualitative interviews. However, we also noted some important areas of divergence. The innovation tournament required substantially less time investment from both the research team and stakeholder participants to complete; however, its electronic platform was expensive, making its overall cost higher than that of observations and interviews. While the two methods generated recommendations in most domains of implementation strategies, observations and interviews generated both more recommendations overall, and more specific recommendations.

Results do not paint a clear picture for the advantage of one method over the other. Rather, researchers must carefully consider the potential information gaps (e.g., strategies relating to adapting and tailoring to context vs. incentives) that may occur from using one participatory design method and weigh the resources available (e.g., money vs. personnel time) to invest in the process when deciding which method to use. It is also important to note that these data do not suggest that the output one participatory design method is more effective than another. An important next step in this line of research will be to share the resultant strategies from each method with stakeholders and evaluate their perception of each strategy's acceptability and feasibility. Whether stakeholder perceptions vary as a function of the chosen participatory design approach it was derived from is an important question to explore in future research. 
There are several reasons why output may differ between the two methods we studied. Observations and interviews allowed for the "up close and personal" examination of the BHT workflow and environment, as well as opportunities for follow-up questions; this may have facilitated obtaining information about adapting and tailoring to context that did not emerge from the innovation tournament. In contrast, the innovation tournament may have been more likely to identify incentive-related strategies because the prompt allowed for emotional "distancing" on the topic from respondents. Specifically, innovation tournament participants were asked to report on what would make data collection for BHTs easier and more motivating broadly (in contrast to speaking specifically to the barriers and facilitators they faced in data collection in the qualitative interview). Participants may have been more likely to think that their colleagues would be motivated by rewards or other incentives to engage in data collection, even if they perceived that they had their own intrinsic motivation to do so, and that the innovation tournament was more likely to elicit this type of information. Participants also may have felt more comfortable sharing ideas about monetary rewards in a more anonymous setting like the innovation tournament, rather than face-to-face during interviews.

Finally, it is possible that the fundamentally different approach to the framing of the inquiry in each approach led to the divergence in findings. During the qualitative interviews, research staff asked openended questions barriers and facilitators to data collection and then deduced potential solutions to the information shared in the generation of implementation strategies. In contrast, the innovation tournament asked respondents to report directly on suggested solutions to the challenge of how to make data collection easier and more motivating and then the research team refined the offered strategies.

Therefore, in many ways, the implementation strategies generated from the observations and interviews incorporate the researchers' experience and expertise in a way that the innovation tournament does not.

There are also likely common disadvantages to both methods that are important to note. In general, people tend to be inaccurate reporters on what would change their own behavior (27). Outputs from both methods may suffer from biased sampling, as all data comes from participants amenable to collaborating with researchers. This may have contributed to the lack of implementation strategies from both methods related to engaging consumers; had we included youth and their families in each participatory design process, results may have differed.

We note several study limitations. First, for both participatory design methods, we had relatively small samples sizes. While our observations and interviews reached thematic saturation, our sample for the innovation tournament was lower than others' (12). BHTs comprise a largely independent contractor workforce and they often work independently at schools, which may have contributed to our difficulty engaging them. Second, we did not evaluate the acceptability, feasibility, or success of any of the identified implementation strategies, so we are unable to evaluate the fit or effectiveness of each participatory design approach, only to highlight points of convergence and divergence. Finally, as noted above, we did not interview youth and families in this study, nor did we invite them to participate in the innovation tournament, which may have led to a gap in identified implementation strategies. This 
underscores the potential for exclusion of select stakeholder groups to greatly impact the output of a participatory design approach.

This study also has some notable strengths. Our use of two separate and blinded research teams to execute each participatory design method concurrently allowed us to be confident in the independence of the findings from each method. Importantly, this study represents the first effort to compare the results that emerge from two methods of participatory design, setting the stage for future research to refine selection and execution of participatory methods to optimize the design of effective implementation strategies.

\section{Conclusions}

This study indicates that two methods of participatory design - observations/interviews and an innovation tournament - yield substantial overlap in the information obtained that can be used for implementation strategy development, but that importantly, there is also unique output generated by each. Given the time and resources required to engage in comprehensive contextual inquiry, selecting the best participatory design approach to inform implementation strategies necessitates that researchers carefully consider what each method offers (strategies relating to adapting and tailoring to context vs. incentives) and weigh the resources available (e.g., personnel time vs. money) to invest in the process. This study advances our understanding of the strengths and limitations of different approaches to participatory design, which is critical for helping implementation researchers and policy makers select the approach best suited to address their implementation question.

\section{Abbreviations}

$\mathrm{EBP}=$ Evidence Based Practice

$\mathrm{BHT}=$ Behavioral Health Technician

$\mathrm{ABA}=$ Applied Behavior Analysis

\section{Declarations}

Ethics approval and consent to participate: All procedures have been approved by the City of Philadelphia and University of Pennsylvania IRB.

Consent for publication: Not applicable

Availability of data and materials: Data will be made available upon request. Requests for access to the data can be sent to the corresponding author

Competing interests: The authors declare that they have no competing interests 
Funding: Funding for this research project is supported by the following grants from NIH: NIMH P50113840 (MPIs: Beidas, Buttenheim, Mandell) and NIDA K23 DA 048167 (PI: Rebecca Stewart).

Authors' contributions: All authors substantially contributed to the conception, design, and analysis of the work. EBH, DSM, and JEB drafted the initial manuscript. EBH, BR, KAZ, and HJN analyzed the data from interviews and observations. JEB, DSM, and RES analyzed data from the innovation tournament. All authors read and approved the final manuscript.

Acknowledgements: The authors gratefully acknowledge that this study would not be possible without our collaborating agencies in the City of Philadelphia. We would also like to thank all of the individuals who participated in the study and all who helped recruit those clinicians, making it possible.

\section{References}

1. Sales AE, Wilson PM, Wensing M, Aarons GA, Armstrong R, Flottorp S, et al. Implementation science and implementation science communications: our aims, scope, and reporting expectations. Implementation Science. 2019,14. https://doi.org/10.1186/s13012-019-0922-2

2. Powell BJ, Beidas RS, Lewis CC, Aarons GA, McMillen JC, Proctor EK, et al. Methods to improve the selection and tailoring of implementation strategies. Journal of Behavioral Health Services and Research. 2017,44(2). https://doi.org/10.1007/s11414-015-9475-6

3. Lane-Fall MB, Curran GM, Beidas RS. Scoping implementation science for the beginner: locating yourself on the "subway line" of translational research. BMC Medical Research Methodology. 2019,19. https://doi.org/10.1186/s12874-019-0783-z

4. Waltz TJ, Powell BJ, Fernández ME, Abadie B, Damschroder LJ. Choosing implementation strategies to address contextual barriers: diversity in recommendations and future directions. Implementation Science. 2019,14(1). https://doi.org/10.1186/s13012-019-0892-4

5. Kok G, Gottlieb NH, Peters GJY, Mullen PD, Parcel GS, Ruiter RAC, et al. A taxonomy of behaviour change methods: an intervention mapping approach. Health Psychology Review. 2016,10(3). https://doi.org/10.1080/17437199.2015.1077155

6. Simonsen J, Robertson T. Routledge international handbook of participatory design. London, UK: Routledge, 2013.

7. Dopp AR, Parisi KE, Munson SA, Lyon AR. A glossary of user-centered design strategies for implementation experts. Translational Behavioral Medicine. 2019,9(6):1057-1064. https://doi.org/10.1093/tbm/iby119

8. Green AE, Fettes DL, Aarons GA. A concept mapping approach to guide and understand dissemination and implementation. Journal of Behavioral Health Services and Research. 2012,39(4). https://doi.org/10.1007/s11414-012-9291-1

9. Fernandez ME, ten Hoor GA, van Lieshout S, Rodriguez SA, Beidas RS, Parcel G, et al. Implementation mapping: using intervention mapping to develop implementation strategies. Frontiers in Public 
Health. 2019,7. https://doi.org/10.3389/fpubh.2019.00158

10. Davis M, Beidas RS. Refining contextual inquiry to maximize generalizability and accelerate the implementation process. Implementation Research and Practice. 2021,2. https://doi.org/10.1177\%2F2633489521994941

11. Ranard BL, Ha YP, Meisel ZF, Asch DA, Hill SS, Becker LB, et al. Crowdsourcing-harnessing the masses to advance health and medicine, a systematic review. Journal of General Internal Medicine. 2014,29. https://doi.org/10.1007/s11606-013-2536-8

12. Terwiesch C, Mehta SJ, Volpp KG. Innovating in health delivery: the Penn medicine innovation tournament. Healthcare. 2013,1. https://doi.org/10.1016/j.hjdsi.2013.05.003

13. Asch DA, Terwiesch C, Mahoney KB, Rosin R. Insourcing health care innovation. New England Journal of Medicine. 2014,370(19). https://doi.org/10.1056/NEJMp1401135

14. Stewart RE, Williams N, Byeon YV, Buttenheim A, Sridharan S, Zentgraf K, et al. The clinician crowdsourcing challenge: using participatory design to seed implementation strategies. Implementation Science. 2019,14(1). https://doi.org/10.1186/s13012-019-0914-2

15. Beidas RS, Volpp KG, Buttenheim AN, Marcus SC, Olfson M, Pellecchia M, et al. Transforming mental health delivery through behavioral economics and implementation science: protocol for three exploratory projects. JMIR Research Protocols. 2019,8(2). https://doi.org/10.2196/12121

16. Azad GF, Locke J, Downey MM, Xie M, Mandell DS. One-to-one assistant engagement in autism support classrooms. Teacher Education and Special Education. 2015,38(4). https://doi.org/10.1177\%2F0888406415603208

17. Lopetegui M, Yen PY, Lai A, Jeffries J, Embi P, Payne P. Time motion studies in healthcare: what are we talking about? Journal of Biomedical Informatics. 2014,49. https://doi.org/10.1016/j.jbi.2014.02.017

18. Nuske HJ, McGhee Hassrick E, Bronstein B, Hauptman L, Aponte C, Levato L, et al. Broken bridgesnew school transitions for students with autism spectrum disorder: a systematic review on difficulties and strategies for success. Autism. 2019,23. https://doi.org/10.1177\%2F1362361318754529

19. Sutton S, French DP, Hennings SJ, Mitchell JO, Wareham NJ, Griffin S, et al. Eliciting salient beliefs in research on the theory of planned behaviour: the effect of question wording. Current Psychology. 2003,22(3). https://doi.org/10.1007/s12144-003-1019-1

20. Potthoff S, Presseau J, Sniehotta FF, Breckons M, Rylance A, Avery L. Exploring the role of competing demands and routines during the implementation of a self-management tool for type 2 diabetes: a theory-based qualitative interview study. BMC Medical Informatics and Decision Making. 2019,19(1). https://doi.org/10.1186/s12911-019-0744-9

21. Michie S, Atkins L, West R. The behaviour change wheel: a guide to designing interventions. London, UK: Silverback Publishing, 2014.

22. Waltz TJ, Powell BJ, Chinman MJ, Smith JL, Matthieu MM, Proctor EK, et al. Expert recommendations for implementing change (ERIC): protocol for a mixed methods study. 
Implementation Science. 2014,9(1). https://doi.org/10.1186/1748-5908-9-39

23. Dillman DA, Phelps G, Tortora R, Swift K, Kohrell J, Berck J, et al. Response rate and measurement differences in mixed-mode surveys using mail, telephone, interactive voice response (IVR) and the Internet. Social Science Research. 2009,38(1). https://doi.org/10.1016/j.ssresearch.2008.03.007

24. Stewart RE, Beidas RS, Last BS, Hoskins K, Byeon YV, Williams NJ, et al. Applying NUDGE to inform design of EBP implementation strategies in community mental health settings. Administration and Policy in Mental Health and Mental Health Services Research. 2021,48(1). https://doi.org/10.1007/s10488-020-01052-z

25. Cidav Z, Mandell D, Pyne J, Beidas R, Curran G, Marcus S. A pragmatic method for costing implementation strategies using time-driven activity-based costing. Implementation Science. 2020,15(1). https://doi.org/10.1186/s13012-020-00993-1

26. Waltz TJ, Powell BJ, Matthieu MM, Damschroder LJ, Chinman MJ, Smith JL, et al. Use of concept mapping to characterize relationships among implementation strategies and assess their feasibility and importance: results from the expert recommendations for implementing change (ERIC) study. Implementation Science. 2015,10(1). https://doi.org/10.1186/s13012-015-0295-0

27. Asch DA, Rosin R. Innovation as discipline, not fad. New England Journal of Medicine. 2015,373(7). https://doi.org/10.1186/s13012-015-0295-0

\section{Supplementary Files}

This is a list of supplementary files associated with this preprint. Click to download.

- STROBEchecklistv4combined.docx 